\title{
IL-17 and IL-22 are associated with protection against human kala azar caused by Leishmania donovani
}

\author{
Maira G.R. Pitta,1,2,3 Audrey Romano,1,2,3 Sandrine Cabantous, 1,2,3 Sandrine Henri,1,2 \\ Awad Hammad, ${ }^{4}$ Bouréma Kouriba, ${ }^{1,2}$ Laurent Argiro, ${ }^{1,2,3}$ Musa el Kheir, ${ }^{4}$ \\ Bruno Bucheton, ${ }^{1,2}$ Charles Mary, ${ }^{1,2,5}$ Sayda Hassan El-Safi, 4 and Alain Dessein 1,2,3,4
}

\begin{abstract}
1INSERM, Unité 906, Marseille, France. ²Faculté de Médecine, Aix-Marseille Université UMR 906, Marseille, France. ${ }^{3}$ FFR88, Institut de Microbiologie de la Méditerranée, Marseille, France. ${ }^{4}$ Faculty of Medicine, Department of Medical Microbiology and Parasitology, University of Khartoum, Khartoum, Sudan. ${ }^{5}$ Assistance Publique, Hôpitaux de Marseille, CHU Timone, Laboratoire de Parasitologie Mycologie, Marseille, France.
\end{abstract}

\begin{abstract}
IL-17 and IL-22 have been shown to increase protection against certain bacteria and fungal pathogens in experimental models. However, no human studies have demonstrated a crucial role of IL-17 and IL-22 in protection against infections. We show here that Leishmania donovani, which can cause the lethal visceral disease Kala Azar (KA), stimulates the differentiation of Th17 cells, which produce IL-17, IL-22, and IFN- $\gamma$. Analysis of Th1, Th2, and Th17 cytokine responses by cultured PBMCs from individuals in a cohort of subjects who developed KA or were protected against KA during a severe outbreak showed that IL-17 and IL-22 were strongly and independently associated with protection against KA. Our results suggest that, along with Th1 cytokines, IL-17 and IL-22 play complementary roles in human protection against KA, and that a defect in Th17 induction may increase the risk of KA.
\end{abstract}

\section{Introduction}

Th17 cells are independently regulated $\mathrm{CD} 4^{+} \mathrm{T}$ cells initially characterized as producing cytokines in the IL-17 family. They are highly proinflammatory and stimulate the production, by endothelial and epithelial cells and monocytes, of cytokines such as IL-6 and TNF and chemokines such as CXCL8 (also known as IL-8), CXCL10 (also known as IP-10), CXCL1, and CXCL6 (1). Th17 cells carry skin and mucosal homing receptors such as CCR6 and CCR4 (2) and recruit neutrophils and monocytes within tissues (3). IL-17 is mostly produced by Th17 and NKT cells (1). IL-22 is also produced by Th17 cells, and to a lesser extent by Th1 and NK cells (1), and is involved in immunity at the epithelium and mucosal surfaces (4). It also promotes the inflammatory response and participates in tissue repair $(5,6)$. The functional IL-22 receptor is expressed on hepatocytes, keratinocytes, and fibroblasts, but not on hematopoietic cells $(4,7)$. Both IL-17 and IL-22 have been shown to increase protection against certain bacteria and fungal pathogens in experimental models $(2,3,8-13)$. However, this protective role has yet to be confirmed in humans. We show here that Leishmania donovani strongly induces Th17 cell differentiation and that IL-17 and IL-22 are strongly and independently associated with resistance to KA.

L. donovani is a protozoan parasite of macrophages responsible for a lethal visceral disease, kala azar (KA). Infected children die due to the multiplication of $L$. donovani in the spleen and liver, associated with splenomegaly, pancytopenia, anemia, and disseminated hemorrhages (14). The protective immune mechanisms operating at early stages of skin and liver infection are not well understood. Most subjects recovering from $L$. donovani infection after drug treatment are protected against repeat infection by Th1-mediated immunity (15). However, one-third of cured patients present subsequent relapses.

Conflict of interest: The authors have declared that no conflict of interest exists. Nonstandard abbreviations used: KA, kala azar; OR, odds ratio.

Citation for this article: J. Clin. Invest. 119:2379-2387 (2009). doi:10.1172/JCI38813.
Neutrophils are a major target of Th17 cells and might be involved in protection against Leishmania infection in mice (16-20). Furthermore, the protection against $L$. donovani transferred with activated dendritic cells in mice is dependent on IL-6, IL-12p40 (21) (a subunit common to IL-12 and IL-23), and IL-23, which are essential for Th17 cell differentiation and maintenance. The levels of both of these cytokines are also particularly high in humans infected with $L$. donovani $(22$, 23). These observations led us to evaluate the possible role of Th17 cells in the protection of humans against KA.

We show here that $L$. donovani induces production of IL-17 and IL-22 by PBMCs from healthy donors. IL-17 was contributed mostly by Th17 cells. Analysis of IL-17 and IL-22 produced by PBMCs of subjects who were recently cured of KA, had developed KA within a few months of cytokine evaluation, or had resisted KA showed independent and strong associations of IL-17 and IL-22 with resistance to KA. This suggests that IL-17 and IL-22 may play complementary roles with Th1 cytokines in human protection against KA. Furthermore, we report that the low IL-17 and IL-22 responses in KA subjects may be related to a defective response to cytokines that initiate the Th17 response.

\section{Results}

L. donovani strongly induces IL-17 and IL-22 in PBMCs from bealthy subjects. PBMCs from the blood of healthy donors were cultured with heat-killed $L$. donovani parasites. Cultures were stimulated with either heat-killed $L$. donovani or with heat-killed $L$. donovani opsonized with $\mathrm{Ab}$ from asymptomatic subjects from regions of endemic infection. In 8 of 10 experiments, we detected IL-17 and IL-22 after 7 to 15 days of culture (Figure 1A). Heat-killed opsonized parasites induced IL-17 and IL-22 responses more efficiently than did heat-killed parasites. L. donovani extracts boosted a secondary response but could not replace the parasite for primary immunization. IL-17 and IL-22 were produced mostly by $\mathrm{CD} 4^{+} \mathrm{T}$ cells, as the $\mathrm{CD} 4^{+} \mathrm{T}$ cells purified from the cultures 5 days after the boost produced large amounts of IL-17 
A

Stimulation

12

None None

OpsLd None

OpsLd OpsLd

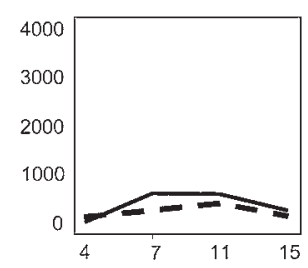

PBMC
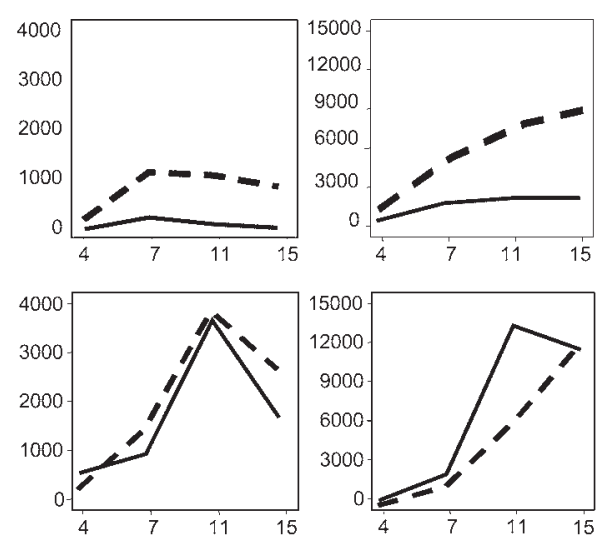

OpsLd $\begin{array}{r}\text { Ld-ext } \\ 10 \mu \mathrm{\mu g} / \mathrm{ml}\end{array}$

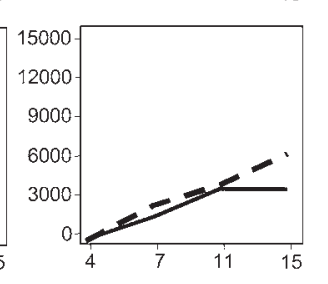

Ld-ext Ld-ext
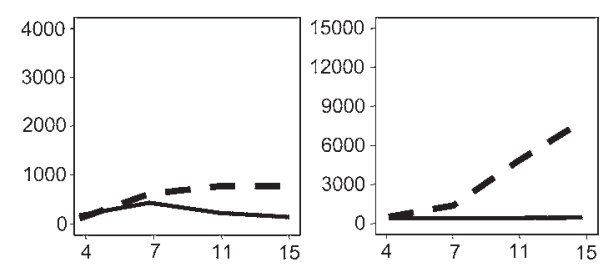

$10 \mu \mathrm{g} / \mathrm{ml} 10 \mu \mathrm{g} / \mathrm{ml} 2000$
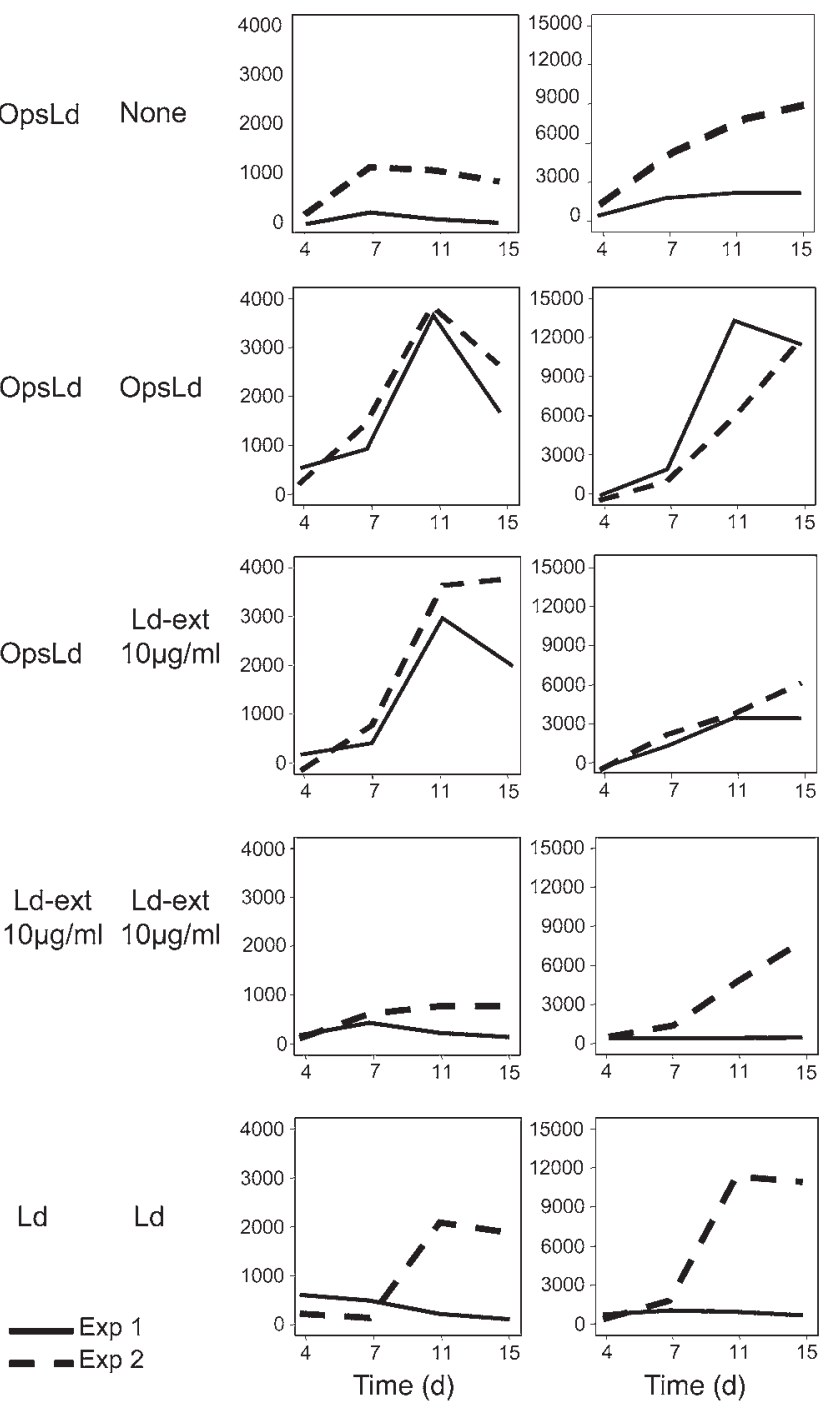

B

Stimulation

1 - 2

None

None

OpsLd

OpsLd

C

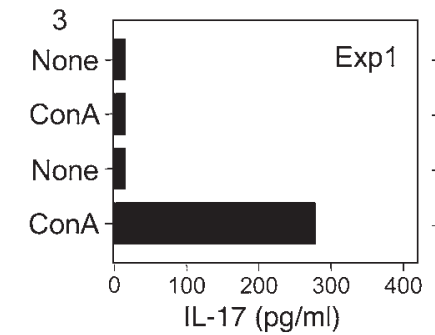

IL-17

$\mathrm{CD4}^{+} \mathrm{T}$ cells

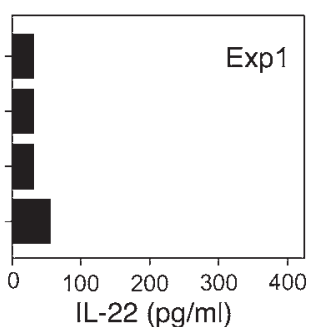

None

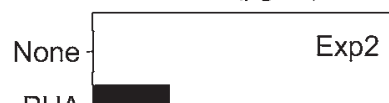

None

OpsLd

OpsLd

PHA

None

PHA

HA
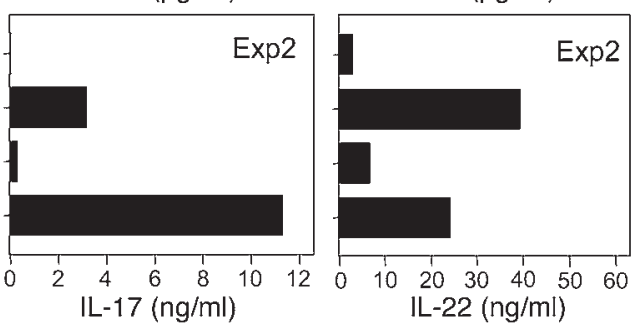

None

None PMA+lono

OpsLd None-

OpsLd PMA+Iono

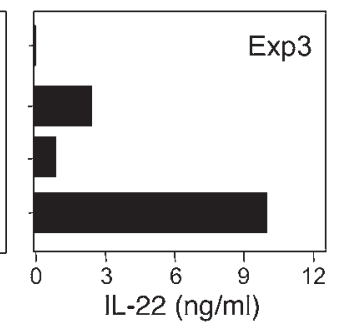

IL-17 $(\mathrm{ng} / \mathrm{ml})$
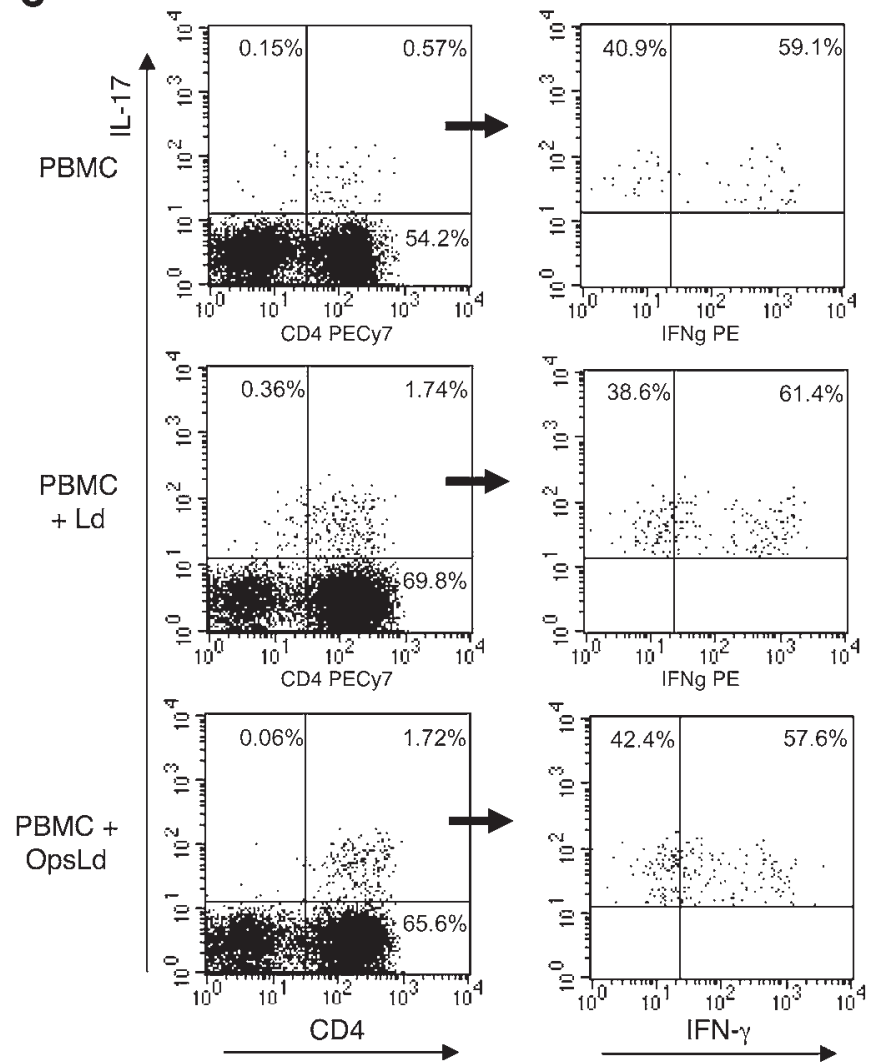


\section{Figure 1}

Induction of IL-17 and IL-22 by L. donovani in cultures of PBMCs from healthy subjects. (A) The production of IL-17 and IL-22 in cultures of PBMCs from healthy donors required priming with whole $L$. donovani parasites. Boosting was achieved using parasites or parasite extracts. Priming (on day 0 ; stimulation 1) and boost (on day 7; stimulation 2) were performed with opsonized (OpsLd) or non-opsonized (Ld) heat-killed L. donovani parasites or parasite extracts (Ld-ext). IL-17 and IL-22 were quantified by ELISA on days 7, 11, and 15. Two representative experiments (Exp 1 and Exp 2) from a series of 10 are shown. (B) IL-17 and IL-22 were produced by $\mathrm{CD} 4^{+} \mathrm{T}$ cells. CD4 ${ }^{+} \mathrm{T}$ cells were purified from the 12-day cultures of PBMCs primed and boosted with OpsLd. Purified $\mathrm{CD}^{+} \mathrm{T}$ cells were then stimulated for 48 hours with PMA plus ionomycin, concanavalin A (ConA), or phytohemagglutinin (PHA). IL-17 and IL-22 were quantified by ELISA. Three independent experiments with cells from different donors are shown. (C) Phenotypic characterization of IL-17+CD4+ $\mathrm{T}$ cells in cultures of normal PBMCs stimulated with OpsLd. Normal PBMCs were primed and boosted with OpsLd. Five days after the second stimulation, cells were stimulated with PMA plus ionomycin plus monensin for 5 hours. The $x$ axis represents the fluorescence intensity after either CD4 labeling or IFN- $\gamma$ labeling. The $y$ axis corresponds to IL-17 labeling. Horizontal and vertical bars define the upper fluorescence intensity observed with labeled control cells. The numbers in each quadrant represent the percentage of cells in that quadrant relative to the total number of cells included in the analysis.

and IL-22 in response to stimulation with concanavalin A, phytohemagglutinin, or ionomycin plus PMA (Figure 1B). Adherent cells produced neither IL-17 nor IL-22 (data not shown). FACS analysis (Figure 1C) showed that stimulation with $L$. donovani increased the proportion of CD4 ${ }^{+} \mathrm{IL}-17^{+}$cells in cultures by 3 - to 70 -fold and that $9 \%$ to $60 \%$ of the induced Th17 cells produced IFN- $\gamma$ (Table 1 ).

$L$. donovani induces $I L-6, I L-1 \beta$, and IL-23 in cultures. Experimental work with human cells has shown that IL-6, TGF- $\beta$, IL-1 $\beta$, IL-23, and possibly IL-21 induce or maintain Th17 cells $(1,24-26)$. IL-1 $\beta$ and IL- 6 but not IL-23 were detected in L. donovani-stimulated cultures of PBMCs from healthy subjects (Figure 2A). IL-23 was produced, together with IL- 6 and IL-1 $\beta$, by L. donovani-stimulated normal blood monocytes (Figure 2B). TGFB mRNA levels in PBMCs were moderately increased by stimulation with $L$. donovani (data not shown). IL-21 was not detected in any culture, including those producing large amounts of IL-17.

Production of Th1 and Th2 cytokines during a KA outbreak. During a 6-year survey of the population of a Sudanese village, we selected a group (gp1) of 87 resistant subjects who did not develop KA either before or after evaluation of their cytokine response, a group (gp2) of 122 subjects who had developed KA during the 24 months preceding cytokine evaluation, and a group (gp3) of 20 subjects who developed KA within 1 to 6 months of evaluation (Table 2). We evaluated Th1, Th2, and Th17 responses in cultures of PBMCs from these 3 groups of subjects.

We first evaluated the Th1 and Th2 cytokines (Figure 3A), which have been shown to be essential for immunity to leishmaniasis in animals (27). IL-4 was undetectable in most cultures and data for this cytokine are therefore not presented; IL-5 and IL-13 were produced in small amounts in most cultures, whereas the levels of IFN- $\gamma$ and IL-12p40 (but not IL-12p70)

\section{Table 1}

were high in the cultures. Nonparametric tests showed that subjects in gp1 produced more TNF $(P<0.001)$, slightly more IL-12p40 $(P=0.03)$, and more IL-10 $(P<0.001)$ than did subjects in $\mathrm{gp} 2$. No significant differences in IL-5 $(P=0.23)$, IL-13 $(P=0.053)$, IFN- $\gamma$ $(P=0.07)$, and IL-12p70 $(P=0.11)$ levels were observed between gp1 and $\mathrm{gp} 2$. Multivariate analysis was carried out because correlations between cytokine levels, age, and sex might have confounded the analysis. Analysis I (Table 3) indicated that TNF levels were higher in gp1 cultures $(P=0.003$; odds ratio $[\mathrm{OR}]=0.23$, CI 0.09-0.60), whereas the highest IFN- $\gamma$ levels were observed in gp 2 cultures $(P=0.016 ; \mathrm{OR}=3, \mathrm{CI} 1.22-7.40)$. Age was a significant confounding variable $(P<0.001)$, whereas sex was not.

We also compared cytokine levels in cultures of cells from gp1 with those in cultures of cells from gp3. Univariate analysis showed that the subjects in gp3 produced less TNF $(P=0.05)$ and less IFN- $\gamma(P=0.03)$ than did subjects in gp1. However, multivariate analysis revealed no significant associations.

$I L-17$ and IL-22 are the cytokines most strongly associated with protection against KA. IL-17 was detectable in $80 \%$ of cultures of cells from gp1 subjects, compared with only $30 \%$ of cell cultures from gp 2 subjects. IL-17 $(P<0.001)$ and IL-22 $(P<0.001)$ levels were higher in cultures of cells from gp1 subjects than in cultures of cells from gp2 subjects (Figure 3A). Furthermore, among the IL-17-positive cultures from gp1 and gp2 subjects, IL-17 levels were higher for subjects in gp1 $(P<0.001)$ than for subjects in $\mathrm{gp} 2$. Almost all cultures produced detectable amounts of IL-22. We carried out a logistic regression analysis to assess the relationship between IL-17 production and the risk of KA. We included IL-17, Th1 cytokines, Th2 cytokines, age, and sex as covariates. The best model for distinguishing between gp1 and gp2 included only IL-17 and age (analysis IIa; Table 3). IL-17 was strongly associated with protection $(P<0.001$; OR $=0.05$, CI $0.12-0.21)$. The risk of past KA in subjects producing low levels of IL-17 was 20 -fold higher than that in subjects producing high levels of IL-17.

We then assessed the relationship between IL-22 and the risk of KA in the presence of Th 1 and Th 2 cytokines, age, and gender. The best model indicated that IL-22 was also significantly associated with resistance to KA. Subjects in the third upper quartile for IL-22 levels were less likely $(P<0.001 ; \mathrm{OR}=0.19$, CI 0.08-0.43) to have presented KA. In this model (analysis IIb, Table 3 ), IFN- $\gamma$ was higher in subjects who had KA $(P=0.003$; OR $=4.70$, CI 4.73-13.10), whereas TNF was lower in the cultures of these subjects as compared with cultures of resistant individuals $(P=0.01 ; \mathrm{OR}=0.27$, CI 0.10-0.75).

These results are illustrated in Figure $3 \mathrm{~B}$, which shows the numbers of resistant subjects and subjects with KA in each IL-17 and

Phenotypic characterization of Th17 cells induced by L. donovani in cultures of PBMCs from healthy donors

\begin{tabular}{|c|c|c|c|c|}
\hline \multirow[t]{2}{*}{ Experiment } & \multicolumn{2}{|c|}{$\%$ IL-17+CD4+ } & \multirow{2}{*}{$\begin{array}{c}\% \text { IFN- } \gamma^{+} \text {in IL-17+CD4+ } \\
\text { Ld-stimulated }\end{array}$} & \multirow{2}{*}{$\begin{array}{c}\% \text { IFN- } \gamma^{+} \text {in } \mathrm{CD}^{+} \\
\text {Ld-stimulated }\end{array}$} \\
\hline & Unstimulated & Ld-stimulated & & \\
\hline 1 & 0.41 & 2.64 & 26.9 & ND \\
\hline 2 & 0.13 & 0.51 & 52.8 & ND \\
\hline 3 & 0.57 & 1.72 & 57.6 & 31.0 \\
\hline $4^{A}$ & 0.07 & 5.14 & 25.0 & 16.9 \\
\hline $5^{A}$ & 0.12 & 0.24 & 26.1 & 24.4 \\
\hline $6^{A}$ & 0.12 & 0.93 & 9.6 & 7.2 \\
\hline
\end{tabular}

Six independent experiments on PBMCs primed and boosted with OpsLd, as described in Figure $1 \mathrm{C}$, are presented. ${ }^{A}$ Cultures supplemented with $10 \mathrm{U} / \mathrm{ml} \mathrm{IL}-2$. ND, not determined. 

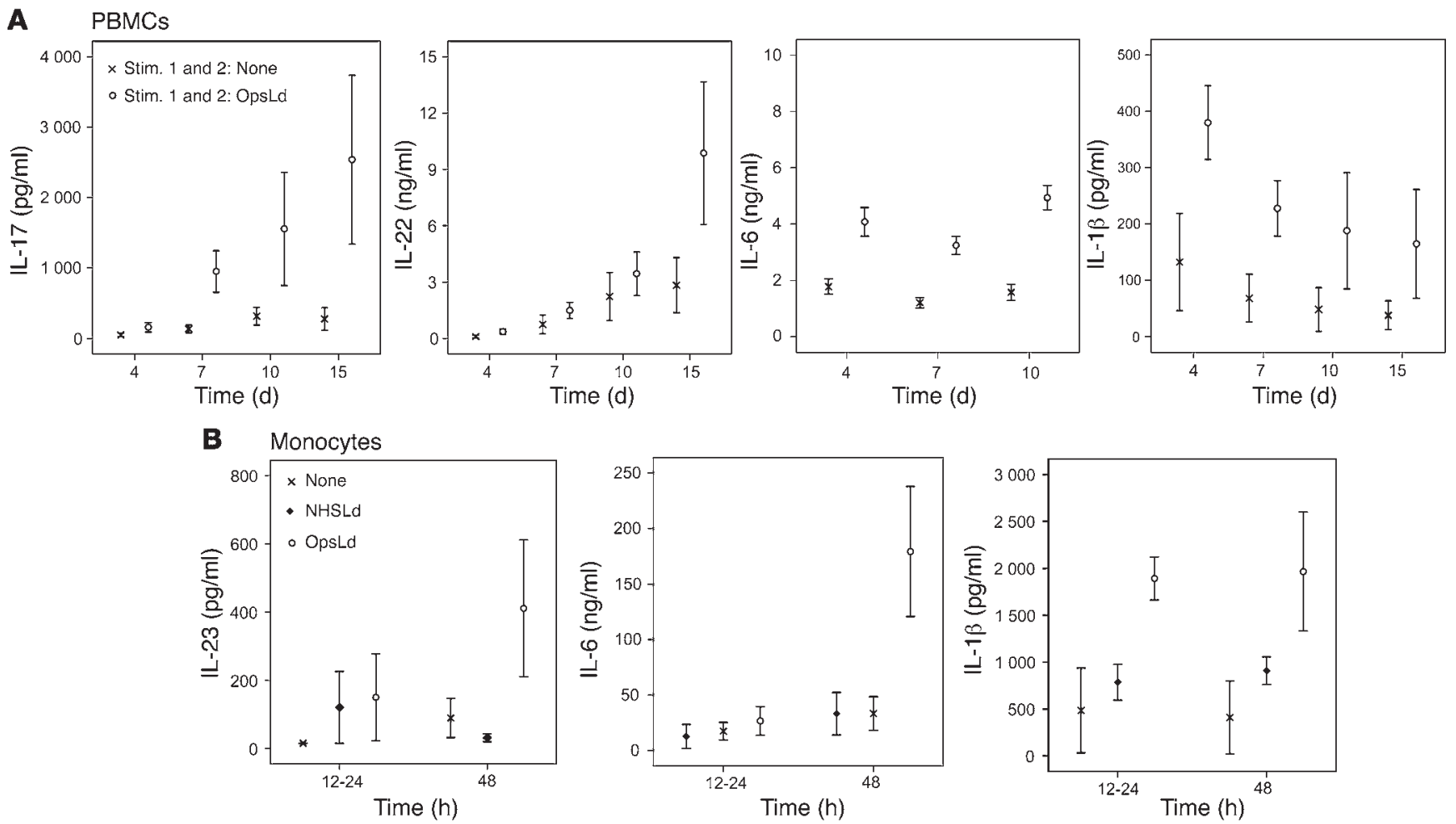

Figure 2

L. donovani induces the production of cytokines that are required for Th17 induction and maintenance. IL-6, IL-23, and IL-1 $\beta$ production in IL-17and IL-22-producing cultures of PBMCs (A) and monocytes (B) from healthy blood donors. PBMCs were primed (day 0 ) and boosted with OpsLd (day 7), as described in the legend of Figure 1. Monocytes were stimulated once with living $L$. donovani that were opsonized with either HIS or NHS. Cytokine levels were determined at the time points indicated. Results represent the arithmetic means of data from 4 independent experiments with PBMCs and 4 experiments with monocytes. The effects of the stimulation and culture duration were tested by linear regression $(P<0.05$ was considered significant). Stimulations had a significant effect on IL-17 $(P=0.003)$, IL-22 $(P=0.05)$, IL-6 $(P<0.001)$, and IL-1 $\beta$ $(P<0.001)$ in cultures of PBMCs and on IL-23 $(P=0.05)$, IL-6 $(P=0.02)$, and IL-1 $\beta(P<0.01)$ in cultures of monocytes. Culture duration had a significant effect $(P<0.01)$ in all cultures excepted for IL-1 $\beta$ (PBMCs and monocytes) and IL-23 (monocytes). Data are means \pm SEM.

IL-22 class. The proportion of resistant subjects was highest in the classes with the highest levels of IL-17 and IL-22. Conversely, the proportion of subjects with KA increased with decreasing IL-17 and IL-22 levels. We investigated the possibility that this result indicated independent contributions of IL-17 and IL-22 to protection rather than a simple correlation between the levels of these 2 cytokines, by carrying out a regression analysis that included both cytokines and age. IL-17 $(P<0.001)$ and IL-22 $(P=0.01)$ were independently associated with resistance (analysis III, Table 3 ). An increase of $1,000 \mathrm{pg} / \mathrm{ml}$ in IL-22 concentration was associated with a 0.66 -fold (CI 0.47-0.91) decrease in the risk of KA, whereas an increase of $100 \mathrm{pg} / \mathrm{ml} \mathrm{IL-17}$ concentration was associated with a 0.34 -fold (CI 0.22-0.52) decrease in the risk of KA. IL-22 and IL-17 levels in cultures varied from 15 to $10,000 \mathrm{pg} / \mathrm{ml}$ and from 15 to $1,000 \mathrm{pg} / \mathrm{ml}$, respectively.

A weak $I L-17$ response was predictive of $K A$. The defect in IL-17 and IL-22 in gp2 may be secondary to multiple changes in the immune cytokine network that could have been activated in patients with KA and persisted after clinical cure. We also evaluated IL-17 and IL-22 production in cultures of PBMCs from gp 3 subjects. These patients produced significantly less IL-17 than did gp1 subjects $(P=0.009)$, as shown by logistic regression analysis (analysis IV in Table 3 ). The production of small amounts of IL-17 in culture was associated with a 13-fold increase $(\mathrm{OR}=0.084, \mathrm{CI} 0.013-0.54)$ in the risk of $\mathrm{KA}$ within 6 months of cytokine evaluation. Once again, IL-17 had the most significant effect, excluding all the Th1 and Th2 cytokines tested from the regression model. We also assessed the effect of IL-22 in the presence of IL-17. We found that both high IL-17 ( $P=0.01 ; \mathrm{OR}=0.32$, CI 0.13-0.77) and high IL-22 $(P=0.044 ; \mathrm{OR}=0.61$, CI 0.38-0.98) levels were associated with a decrease in the risk of KA (analysis V in Table 3). The ORs obtained were similar to those obtained for comparisons of the cytokine responses of gp1 and gp2 subjects.

Defective response to Th17 regulatory cytokines in subjects with $K A$. Because IL-1 $\beta$, IL-6, IL-21, and IL-23 are required for the induction and/or maintenance of the Th17 response $(1,24)$, we evaluated the levels of these cytokines in cultures of cells from the subjects studied (Figure 4). IL-21 was undetectable in all cultures, whereas IL-6 $(P<0.001)$ and IL-1 $\beta(P<0.001)$ levels were significantly higher in cultures of cells from gp1 subjects than in cultures of cells from gp 2 subjects. No significant differences in IL-23 levels were observed in between study groups (Figure 4A).

We then tried to determine which of these 3 cytokines was the most strongly associated with IL-17 and IL-22 levels. We found that IL-23 was the most strongly associated with IL-17 $(P<0.001$; $\mathrm{OR}=5.2$, CI 2.3-11.7) and excluded IL-1 $\beta$ and IL- 6 from the regression model. A similar analysis for IL-22 showed that IL-22 production was more closely associated with IL-6 $(P<0.001$; OR $=1.13$, CI 1.31-1.52). These results suggested that differences in IL-23 and IL-6 levels may determine IL-17 and IL-22 levels. We then plotted 


\section{Table 2}

Immunological, parasitological, and clinical criteria for the selection of study subjects

\begin{tabular}{|c|c|c|c|c|c|c|}
\hline & $n$ & $\mathrm{Age}^{A}$ & $\begin{array}{c}\text { Gender } \\
\text { (male/female) }\end{array}$ & $\begin{array}{c}\text { Western } \\
\text { blot }^{B}\end{array}$ & IFN- $\gamma^{+}$ & Selection criteria \\
\hline gp1 & 87 & $32 \pm 18$ & $45 / 42$ & $92 \%$ & $100 \%$ & $\begin{array}{l}\text { Positive Western blot, IFN- } \gamma \text { in cultures, and no infection with KA } \\
\text { during the entire study }\end{array}$ \\
\hline gp2 & 122 & $16 \pm 9$ & $69 / 53$ & $99 \%$ & $83 \%$ & $\begin{array}{l}\text { KA (Leishmania parasites in lymph nodes, and clinical symptoms) } \\
\text { Successfully cured 6-24 months before the study }\end{array}$ \\
\hline gp3 & 20 & $16 \pm 10$ & $5 / 15$ & $89 \%$ & $84 \%$ & $\begin{array}{l}\text { Positive Western blot and/or IFN- } \gamma \text {, and infection with KA within } \\
6 \text { months following cytokine evaluation }\end{array}$ \\
\hline
\end{tabular}

AData are the arithmetic mean \pm SD. ${ }^{B}$ Stained positive in the 14- to $16-\mathrm{kDa}$ band of a Western blot.

IL-17 and IL-22 levels as a function of IL-23, IL-6, and IL-1 $\beta$ levels in cultures, assuming a similar relationship between the levels of IL-17 or IL-22 and regulatory cytokines in gp1 subjects and gp2 subjects (Figure 4B). Surprisingly, cultured cells from gp2 subjects produced much lower levels of IL-17 for the same amounts of the 3 regulatory cytokines. Cultures of cells from gp1 subjects showed a clear correlation of IL-17 concentration with the concentration of all 3 regulatory cytokines. In contrast, we did not observe such a correlation between IL-17 levels and IL-1 $\beta$ and IL- 6 in gp 2 cultures. This suggests that a defect in the Th17 differentiation pathway may affect the response to these 2 cytokines in subjects susceptible to KA. The picture was less clear in a similar analysis for IL-22: the IL-22 response increased with IL-23 and IL- 6 concentrations. However, this increase was less marked in gp2 subjects than in gp1 subjects.

\section{Discussion}

Our studies with PBMCs from healthy individuals and from subjects exposed to L. donovani showed that Leishmania strongly induced IL-17 and IL-22. This conclusion was further supported by the presence in $L$. donovani-stimulated cultures of IL-1 $\beta$, IL-23, and IL-6, the key cytokines for the induction and maintenance of the Th17 response $(1,24,26,28,29)$. No IL-21 was detected, consistent with other reports suggesting that IL-21 may be dispensable for human Th17 induction $(30,31)$. The heat-killed or parasite extracts used to induce cytokine responses in PBMCs is mostly confined to promastigotes. The stimulation of monocyte cultures from healthy patients with either $L$. donovani extract or living parasites that progressed to the amastigote stage yielded similar results. IL-17 was produced in cultures of PBMCs from healthy donors by Th17 and Th1/17 cells, as has also been reported in other human studies (32). We cannot exclude that IL-17 may also be produced by NKT cells $(1,33)$ and $\gamma / \delta$ T cells $(1,4,34)$, and IL-22 may also be secreted by Th1 and NK cells $(1,7)$. It has been discussed that Th $1 / 17$ may represent an intermediate stage of differentiation between Th17 and Th1 cells driven by IL-12 (32). human Th17 and human Th1/17 cells transcribe the genes encoding Tbet and ROR $\gamma(t)(32,35)$, the master regulators of Th1 and Th17 differentiation, respectively.

The lower production of IL-17 and IL-22 in gp2 subjects might have resulted from alterations of the cytokine network that had occurred during the infection and persisted after clinical cure. To rule out this possibility, we also evaluated IL-17 and IL-22 in gp3 subjects. The comparison of gp1 and gp 3 showed that low IL-17 and IL-22 were predictive of KA.

IL-17 and IL-22 may contribute to protective immunity to $L$. donovani in several ways. The insect vector injects Leishmania into human skin and the parasite then migrates to the liver, where it multiplies
(14). Work in infected animals suggests that neutrophils could be involved in controlling the early stages of Leishmania infection (16-20) and massively infiltrate the skin and liver granulomas that form around Leishmania at early stages of infection. These results have not, however, been confirmed in all studies. The L. donovani granuloma likely contain IL-17- and IL-22-producing T cells because Th17 cells, even after prolonged antigen exposure, carry the skin and mucosal tissue homing receptors CCR6 and CCR4 $(2,36)$. IL-17 stimulates the production of CSF (GM-CSF and G-CSF), which increases the production of neutrophils, monocytes, and CXCL chemokines such as CXCL8 (IL-8), CXCL1, and CXCL6 (GCP2), which serve as potent chemoattractants for neutrophils or CXCL10 (IP10), which acts as a chemoattractant for Th1 cells $(1,7)$. IL-17 also increases the production of IL- 6 , which has proinflammatory and regulatory effects on the immune response (1). IL-22 increases the production of proinflammatory molecules, such as the S-100A proteins and CXCL5 (6). IL-17 and IL-22 synergistically increase the production of antimicrobial peptides, such as $\beta$-defensins, by epithelial cells (37). Finally, IL-22 is involved in epithelial repair (6) and liver protection (38) in chronic infections. Both the increases in epithelial protective barrier function and the recruitment of inflammatory cells, including neutrophils, to the skin and liver, could contribute to protection against $L$. donovani. Work in animals infected with other pathogens has provided support for the view that Th17 cells, IL-17, and IL-22 may be essential for immunity to certain infections (2, 3, 9-13). In these experimental models, neutrophils are important, both as effectors and as modulators of the immune response via secreted molecules such as IL-12. Large numbers of Th17 cells were shown to infiltrate Mycobacterium tuberculosis granuloma in vaccinated mice (3), the mucosal sites of Klebsiella pneumoniae infection in the lung (39, 40 ), and the colon of Citrobacter rodentium-infected mice (41). IL-23 and IL-17 are essential for the expression of full immunity in these experimental models $(3,39,41)$. Finally, Th17 may bridge the gap between innate and adaptive immunity. Th17 cells are required for a recall response of mice vaccinated with $M$. tuberculosis antigen, as they promote the recruitment of Th 1 in the lungs by stimulating the release of mediators such as CXCL10 (3). Th17 cells could play the same role in $L$. donovani infections. Naturally resistant subjects with enhanced IL-17 and IL-22 responses would thus react more rapidly to $L$. donovani, attracting strong effectors of innate immunity and recruiting Th1 cells to tissues. These cells would in turn enhance the microbicidal activity of phagocytes. Thus, Th17 and Th1 cells may play complementary roles in protection against $L$. donovani, with both being required for complete protection. In this association between Th1 and Th17 responses, Th1 cells may play the important role of downregulating the Th17 response after the infection is controlled, 
A
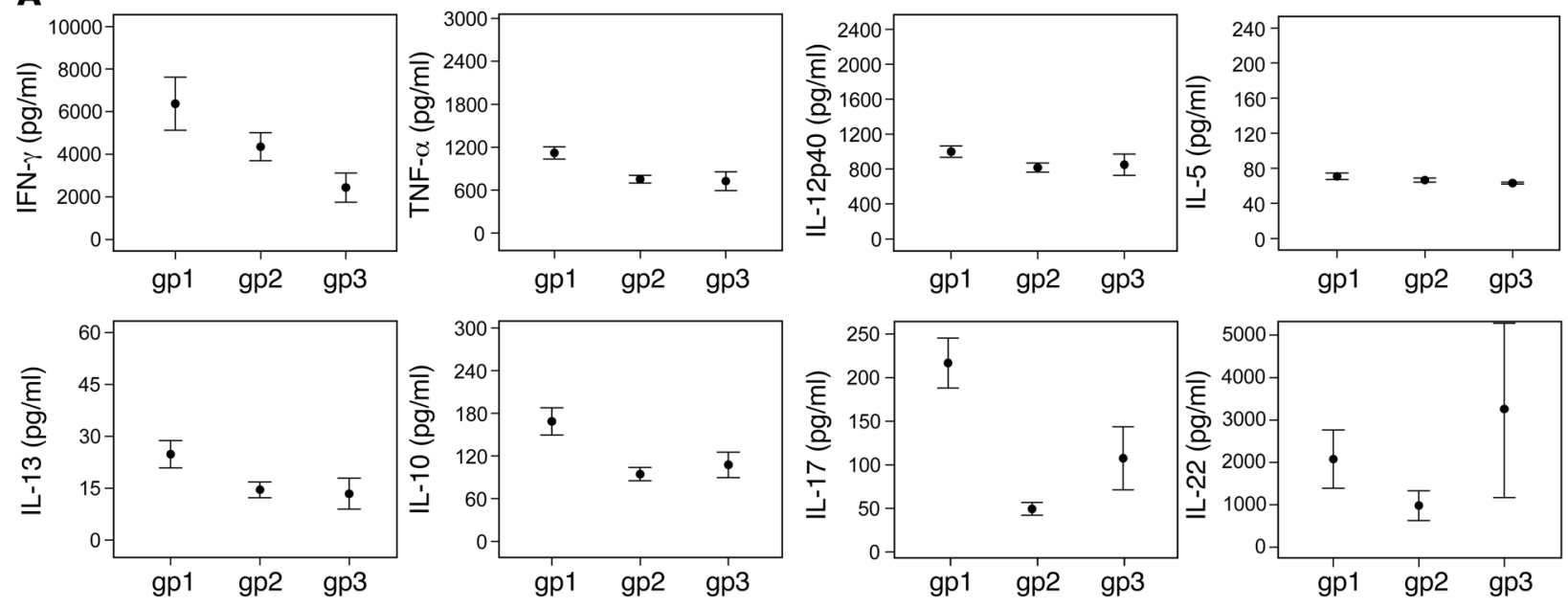

B
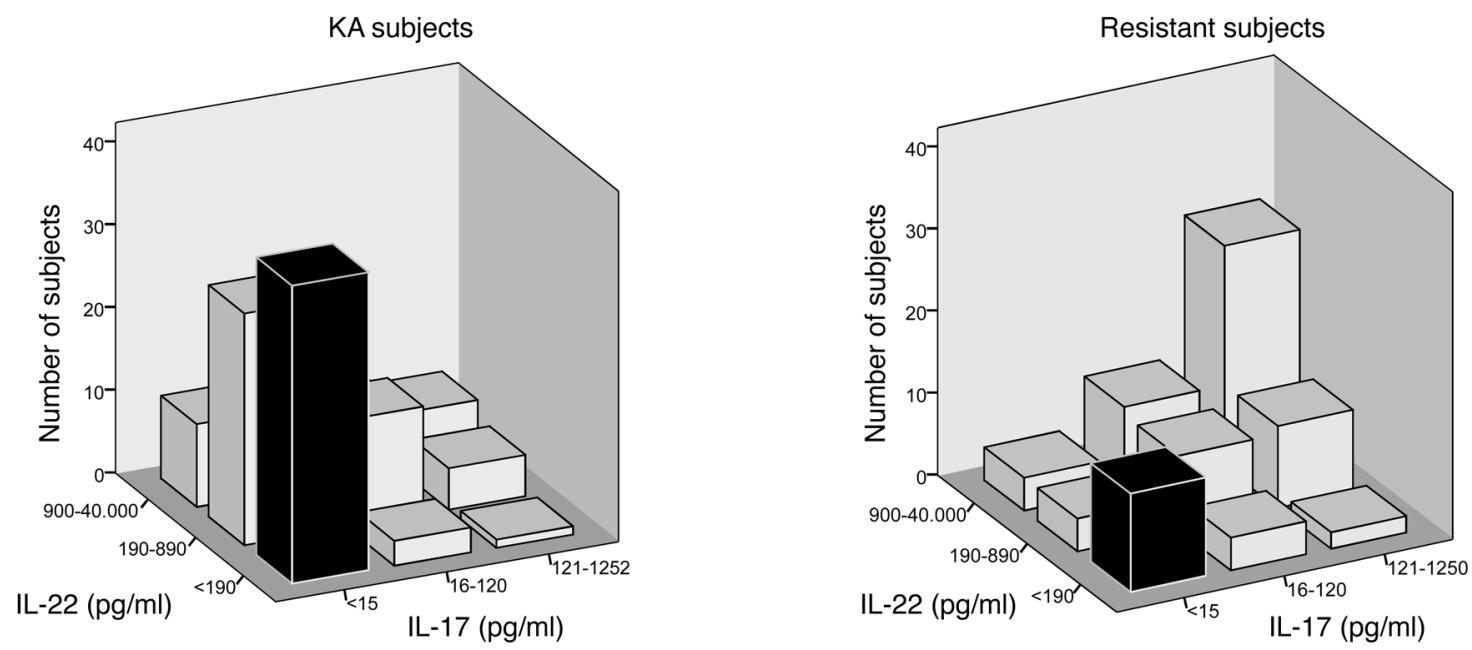

Figure 3

Resistance to KA is associated with enhanced IL-17 and IL-22 responses. (A) IL-17, IL-22, and Th1 and Th2 cytokine production by PBMCs from gp1, gp2, and gp3 subjects. Cytokines were quantified by ELISA in supernatants of PBMCs stimulated with $10 \mu \mathrm{g} / \mathrm{ml}$ of $L$. donovani extracts, as indicated in the Methods. IL-4 was undetectable or present in small amounts in all cultures. The data presented are the arithmetic means \pm SEM. Cytokine scales ( $y$ axes) were set to allow for a 5 -fold increase in the mean levels above those of the group with the lowest levels to be represented. (B) Resistance to KA was associated with high levels of both IL-22 and IL-17, whereas KA was associated with low levels of these cytokines. IL-17 and IL-22 levels were each assigned to 3 classes of equal size. The 3 classes for IL-17 were $\leq 15,16-120$, and 121-1,250 pg/ml. The 3 classes for IL-22 were <190, 190-890, and 900-40,000 pg/ml. Dark bars represent subjects with the lowest IL-17 and IL-22 levels. Data analysis is presented in Table 3 .

thereby preventing tissue damage resulting from the out-of-control expansion of the Th17 cell population.

We explored the mechanisms that may have accounted for the weak IL-22 and IL-17 responses in KA subjects. The IL-22 response induced by $L$. donovani in our cultures was more dependent on IL-6 than on IL-23, and conversely the IL-17 response was more dependent on IL-23 than on IL-6. Other studies have reported different cytokine requirements for IL-22 and IL-17 production, with IL-22 production dependent on IL- 6 but not on TGF- $\beta$, and with IL-17 production dependent on TGF- $\beta$ and IL- 6 (42). IL-23 is required to maintain the Th17 response $(26,43)$. The lower level of production of IL- 6 and IL-23 in cultures from subjects with KA may have contributed to the weaker IL-17 and IL-22 responses of these subjects. Nevertheless, IL-6 is also produced by Th17 cells (1), and lower levels of IL- 6 production in cultures may be a consequence rather than a cause of the smaller number of Th17 cells. Furthermore, although certain cultures of cells from subjects with KA displayed high levels of IL-6, IL-1 $\beta$, and IL-23 production, IL-17 levels remained low, suggesting a poor $\mathrm{T}$ cell response from KA subjects to IL- 6 and IL-1 $\beta$. This defective response to regulatory cytokines was more marked with IL-17 than with IL-22, probably because IL-22 was also produced by Th1 cells (7). Our observations suggest a defect rendering $T$ cells less responsive to key regulators, such as IL-1 $\beta$ and IL-6, that are crucial for Th17 induction.

In conclusion, we show here that $L$. donovani strongly induces IL-17 and IL-22 responses. We have presented evidence that IL-17 and IL-22 play complementary roles in natural protection against $L$. donovani. This view is consistent with the effects of Th17 on monocytes and neutrophils and the ability of Th17 to recruit Th1 cells to the granuloma. In addition, IL-22 and IL-17 promote tissue repair and strengthen epithelial barriers, thereby contributing to immunosur- 
Table 3

Logistic regression analysis of IL-17, IL-22, and Th1 and Th2 cytokines in the risk of KA

\begin{tabular}{|c|c|c|c|c|c|c|}
\hline Analysis & Tested covariates & Covariate in the model & $\operatorname{Class}^{A}(p g / m l)$ & $P$ & $\mathbf{O R}$ & Cl \\
\hline \multicolumn{7}{|c|}{ Comparison: gp1 and gp2 } \\
\hline \multirow[t]{3}{*}{ I } & Th1 and Th2 cytokines & IFN- $\gamma$ & 910 & 0.016 & 3 & $1.22-7.40$ \\
\hline & & TNF & 460 & 0.003 & 0.23 & $0.09-0.60$ \\
\hline & & Age & & $<0.001$ & 0.92 & $0.89-0.94$ \\
\hline \multirow[t]{2}{*}{ Ila } & Th1 and Th2 cytokines and IL-17 & IL-17 & 120 & $<0.001$ & 0.05 & $0.12-0.21$ \\
\hline & & Age & & $<0.001$ & 0.89 & $0.84-0.94$ \\
\hline \multirow[t]{3}{*}{ IIb } & Th1 and Th2 cytokines and IL-22 & IL-22 & 890 & $<0.001$ & 0.19 & $0.08-0.43$ \\
\hline & & IFN- $\gamma$ & 910 & 0.003 & 4.70 & $4.73-13.10$ \\
\hline & & TNF & 460 & 0.01 & 0.27 & $0.10-0.75$ \\
\hline \multirow[t]{4}{*}{ III } & Th1 and Th2 cytokines, IL-17, and IL-22 & IL-17B & & $<0.001$ & 0.34 & $0.22-0.52$ \\
\hline & & IL-22B & & 0.01 & 0.66 & $0.47-0.92$ \\
\hline & & IL-17 and IL-22 & & 0.003 & & \\
\hline & & Age & & $<0.001$ & 0.90 & $0.86-0.97$ \\
\hline \multicolumn{7}{|c|}{ Comparison: gp1 and gp3 } \\
\hline \multirow[t]{2}{*}{ IV } & Th1 and Th2 cytokines and IL-17 & IL-17 & 15 & 0.009 & 0.08 & $0.013-0.54$ \\
\hline & & Age & & 0.01 & 0.91 & $0.84-0.96$ \\
\hline \multirow[t]{4}{*}{ V } & Th1 and Th2 cytokines, IL-17, and IL-22 & IL-17B & & 0.011 & 0.32 & $0.13-0.77$ \\
\hline & & $\mathrm{IL}-22^{\mathrm{B}}$ & & 0.044 & 0.61 & $0.38-0.98$ \\
\hline & & IL-17 and IL-22 & & 0.01 & & \\
\hline & & Age & & 0.002 & 0.91 & $0.86-0.97$ \\
\hline
\end{tabular}

Analysis I compared Th1 and Th2 cytokines in the risk of KA. Analysis Ila added IL-17 to the same analysis and showed the strongest association with protection against KA. Analysis IIb compared IL-22 and Th1 and Th2 cytokines in the risk of KA. Analysis III found that both IL-22 and IL-17 were independently associated with resistance to KA. Analysis IV found that IL-17 was associated with protection against KA that occurred in the months following cytokine evaluation. Analysis $V$ found that both IL-17 and IL-22 were independently associated with protection against KA that occurred in the months following cytokine evaluation. An OR greater than 1 indicates an increased risk with an increase of the covariate; an OR less than 1 indicates a decreased risk with an increase of the covariate. ${ }^{A}$ When the covariates were used as a qualitative variable, the indicated cytokine levels were used to define the covariate class. ${ }^{B} \mathrm{IL}-17$ and IL-22 were treated as quantitative variables. In order to yield a meaningful OR, IL-17 and IL-22 levels were multiplied by 0.01 and 0.001 , respectively (e.g., in analysis $\mathrm{V}$, an increase of $100 \mathrm{pg} / \mathrm{ml}$ for $\mathrm{IL}-17$ and $1,000 \mathrm{pg} / \mathrm{ml}$ for IL-22 corresponded to a reduction of 0.32 and 0.61 , respectively, in the risk of KA).

veillance. Our work also provides new insight into possible alterations to the immune defenses of subjects with KA. These findings should stimulate the development of new strategies for preventing this severe and devastating parasitic disease responsible for the deaths of large numbers of people in countries in which it is endemic.

\section{Methods}

Study groups. The study groups (Table 2) were recruited from subjects living in Barbar el Fugarra in the eastern part of Sudan $(44,45)$, where we carried out a 6-year longitudinal study on 1,600 subjects during a severe outbreak of KA caused by $L$. donovani. Serological and clinical evaluations were carried out at 16-month intervals. Serological evaluation was performed by ELISA and Western blotting (recognition of the 14- to $16-\mathrm{kDa}$ Leishmania-specific band). About one-third of the villagers had parasites in lymph nodes and clinical symptoms of KA and had been treated with meglumine antimoniate (Glucantime) or sodium stibogluconate (Pentostam). Cytokine production was assessed in 229 selected subjects at the peak of the outbreak; 107 of these subjects had not presented clinical KA but were seropositive (positive by ELISA and Western blot analysis) and produced IFN- $\gamma$ in 120-hour PBMC cultures stimulated with $L$. donovani extracts. Eighty-seven of these 107 seropositive IFN- $\gamma^{+}$subjects did not develop KA at any time during the 6 years of the study (which continued for 2 years after cytokine evaluation). These patients were classified as resistant (gp1). Twenty of the 107 seropositive IFN- $\gamma^{+}$subjects presented KA within 6 months of cytokine evaluation (gp3). The remaining 122 subjects had developed KA in the 2 years before the evaluation (gp2). These 122 subjects had been treated with drugs and successfully cured 6 to 24 months before the cytokine study.
The study was approved by the ethics committee of the University of Khartoum Faculty of Medicine. Informed consent was obtained from all patients. The study was also approved by the village committee.

Preparation of L. donovani antigens. The L. donovani strain MHOM/SD/98/ LEM 3566 was isolated from a patient with KA in the village (46) and maintained in promastigote form by in vitro culture at $24^{\circ} \mathrm{C}$ in RPMI- 1640 medium (Invitrogen) supplemented with 10\% FBS (Cambrex). Soluble L. donovani antigens were prepared as described elsewhere (47).

Parasite opsonization. Serum samples from healthy white donors from the blood bank in Marseille (Etablissement Français du Sang) (normal human serum [NHS]) and pools of human immune serum (HIS) from subjects living in Barbar were heated at $56^{\circ} \mathrm{C}$ for 45 minutes and centrifuged $(12,000 \mathrm{~g}$, 10 minutes). Promastigotes $\left(5 \times 10^{7} / \mathrm{ml}\right)$ were incubated with $10 \% \mathrm{NHS}$ or HIS for 90 minutes at $4^{\circ} \mathrm{C}$. The parasites were then killed by heating at $50^{\circ} \mathrm{C}$ for 3 minutes, washed 3 times in cold culture medium and added to the cultures. The parasites that reacted with HIS and NHS will be referred to hereafter as "OpsLd" and "NHSLd," respectively.

Cell purification and cell cultures. PBMCs were separated from blood from the Marseille blood bank (Etablissement Français du Sang) or from Sudanese patients, as indicated in the Figure 3 legend (47). PBMCs from KA patients $\left(2 \times 10^{6} \mathrm{cell} / \mathrm{ml}\right)$ were stimulated with $10 \mu \mathrm{g} / \mathrm{ml}$ of $L$. donovani antigens (referred to as "Ld-ext"). PBMCs from healthy donors were stimulated with $10^{6} \mathrm{OpsLd}$ or with $10 \mu \mathrm{g} / \mathrm{ml} \mathrm{Ld}$-ext and again on day 7 with the same stimulations. Monocytes and $\mathrm{CD}^{+} \mathrm{T}$ cells were purified (>98\%) by magnetic cell sorting (Miltenyi Biotec) from PBMC cultures. Monocytes $\left(8 \times 10^{5} \mathrm{cells} / 500 \mu \mathrm{l}\right)$, purified on day 0 from PBMC cultures, were stimulated with $10^{6} \mathrm{OpsLd}$ or NHSLd. CD4 $4^{+} \mathrm{T}$ cells $\left(2 \times 10^{5}\right.$ cells $\left./ 200 \mu \mathrm{l}\right)$, purified 
A
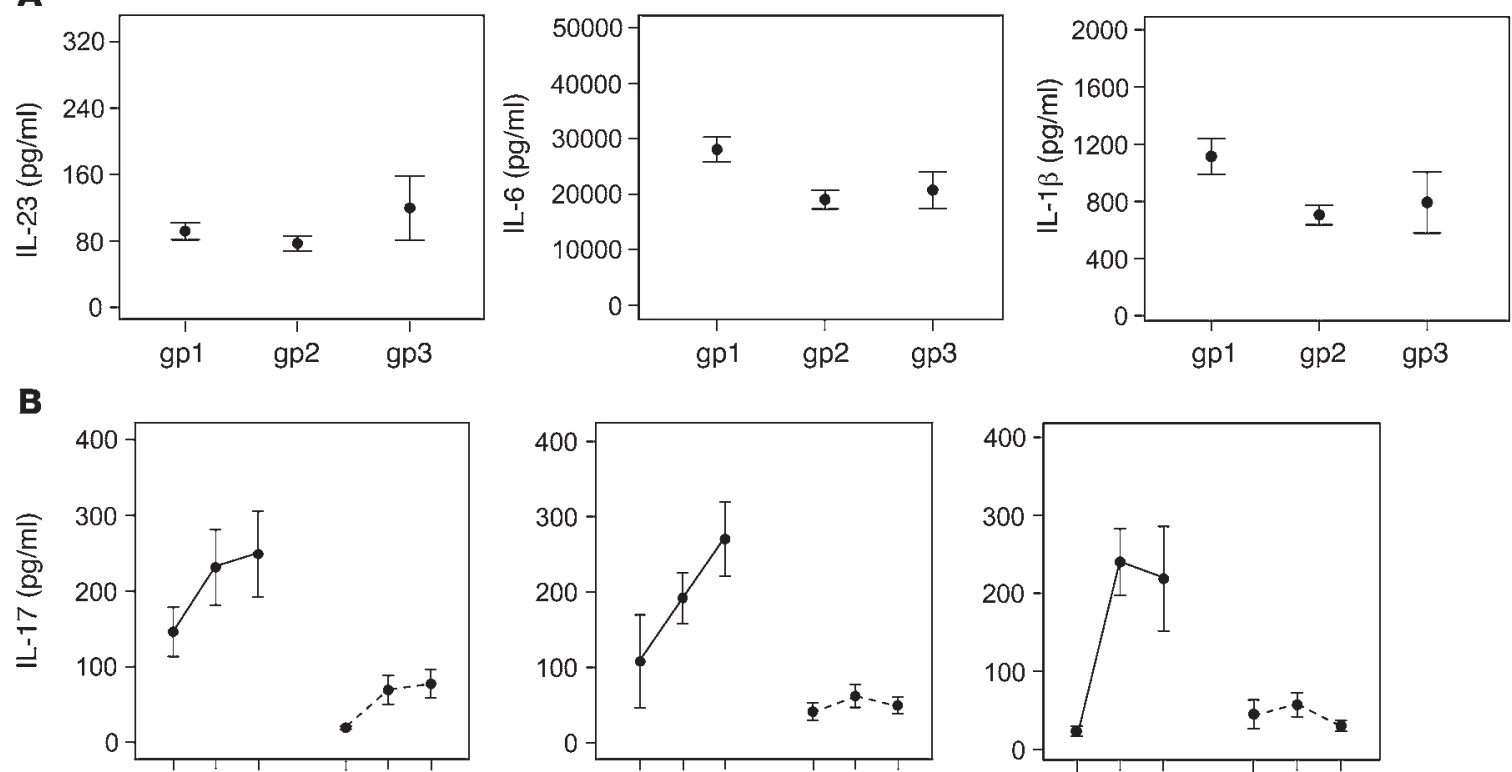

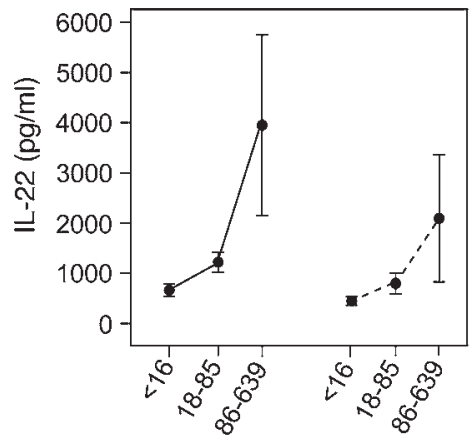

$\mathrm{IL}-23(\mathrm{pg} / \mathrm{ml})$

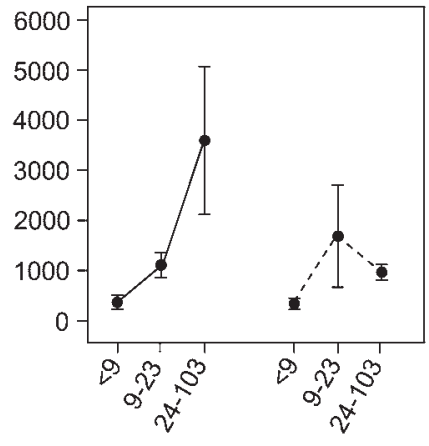

IL-6 (pg/ml)

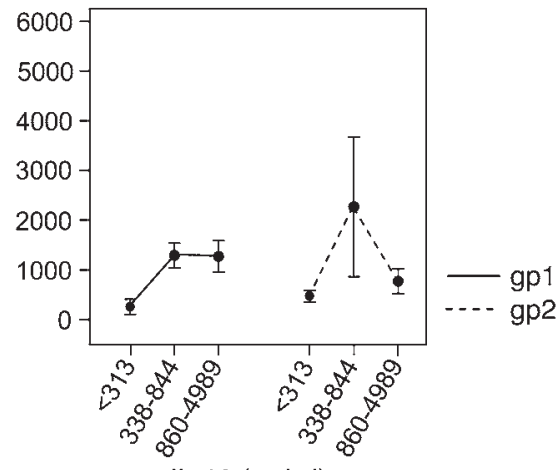

$\mathrm{IL}-1 \beta(\mathrm{pg} / \mathrm{ml})$

\section{Figure 4}

IL-23, IL-6, and IL-1 $\beta$ production in cultures of PBMCs from subjects from the 3 clinical groups. (A) IL- 6 and IL-1 $\beta$ levels were higher in cultures of PBMCs from gp1 subjects than in those of cells from gp2 subjects, whereas no difference was observed for IL-23. Cultures were stimulated as described in Figure 2A. (B) PBMCs from KA subjects produced small amounts of IL-17 and IL-22, even in the presence of normal to high levels of the regulatory cytokines IL-6, IL-1 $\beta$, and IL-23. IL-23, IL-6, and IL-1 $\beta$ levels in cultures (from gp1 and gp2) were assigned to 3 classes of equal size, and IL-17 and IL-22 levels are presented for each cytokine class. A nonparametric Kruskal-Wallis test showed a significant increase of IL-17 $(P<0.03)$ in increasing IL-23, IL-6, and IL-1 $\beta$ classes in cultures of PBMCs from resistant subjects. No significant increase of IL-17 was observed with increasing concentrations of IL- 6 and IL-1 $\beta$ in cultures of PBMCs from gp2 subjects, whereas IL-17 did increase $(P<0.001)$ with increasing IL-23 concentrations in the same cultures. IL-22 increased $(P<0.05)$ with increasing IL-23, IL-6, and IL-1 $\beta$ concentrations in cultures of PBMCs from gp1 and gp2 subjects. The data presented are the arithmetic means \pm SEM.

on day 15 from PBMC cultures, were stimulated with PMA $(100 \mathrm{ng} / \mathrm{ml})$ plus ionomycin $(1 \mu \mathrm{g} / \mathrm{ml})$, concanavalin A $(5 \mu \mathrm{g} / \mathrm{ml})$, or phytohemaggluti$\operatorname{nin}(5 \mu \mathrm{g} / \mathrm{ml})$ (Sigma-Aldrich). Resting cells were used as controls.

Intracellular cytokine staining. Cells were stained for surface markers with PE-Cy7-conjugated anti-CD4 (eBiosciences) antibodies. The labeled cells were pulsed with $100 \mathrm{ng} / \mathrm{ml}$ PMA, $1 \mu \mathrm{g} / \mathrm{ml}$ ionomycin and monensin (BD GolgiStop) for 5 hours. Cells were then fixed and permeabilized with BD Cytofix/Cytoperm according to the manufacturer's instructions and incubated with Alexa Fluor 647-conjugated anti-IL-17 and PE-conjugated anti-IFN- $\gamma$ (eBiosciences) antibodies. Isotype controls were obtained from the corresponding manufacturers. Data were analyzed on a FACSCalibur flow cytometer, using CellQuest software (BD Biosciences).

Cytokine titration. Cytokines in the supernatants of cultures were assayed with ELISA kits according to the manufacturer's instructions. In cultures from endemic subjects, IL-6 (Diaclone), IL-17A (R\&D Systems), IL-22 (R\&D Systems), IL-21 (eBiosciences), IL-5 (BD Biosciences), and IFN- $\gamma$ (BD Biosciences) levels were measured at 120 hours. IL-1 $\beta$ (R\&D Systems), IL-4 (Abcys), IL-23 (eBioscience), IL-12p40 (BD Biosciences), IL-13 (Diaclone), and IL-10 (BD Biosciences) and TNF (BD Biosciences) were quantified at 48 hours. The supernatants of PBMCs, $C D 4^{+} \mathrm{T}$ cells, or monocytes obtained from healthy subjects were collected at the times indicated in the figures. The lower limits of detection for the ELISA analyses were as follows: $0.78 \mathrm{pg} / \mathrm{ml}$ for IL-13; $1.56 \mathrm{pg} / \mathrm{ml}$ for IL-6; $1.9 \mathrm{pg} / \mathrm{ml}$ for IL- $1 \beta ; 5 \mathrm{pg} / \mathrm{ml}$ for IFN- $\gamma ; 7.8 \mathrm{pg} / \mathrm{ml}$ for IL-23, IL-4, TNF, and IL-10; $15 \mathrm{pg} / \mathrm{ml}$ for IL-12p40, IL-17A, and IL-22; and $32.5 \mathrm{pg} / \mathrm{ml}$ for IL-21.

Statistics. The statistical methods that were used are indicated in the tables and figures. Univariate comparisons were carried out using nonparametric tests (Mann-Whitney tests). $P<0.01$ was considered as indi- 
cating a significant association and $P<0.05$ a suggestive association. The association of cytokines with the risk of KA was also evaluated by logistic regression using clinical binary traits (resistant versus previous KA; resistant versus subsequent KA). The covariates were age, sex, and cytokines. Cytokines were assigned to 3 classes (1,2, and 3 ) of equal size and treated as binary variables ( 1 versus $2+3$, and $1+2$ versus 3 ). The cytokine levels defining the classes significantly associated with the phenotype in the regression analysis are indicated in the Table 3 legend. When IL-17 and IL-22 were tested jointly in the model, they were entered as linear variables, making it possible to account for the correlation between the levels of these 2 cytokines. Linear regression was used to evaluate the associations between regulatory cytokines (IL-6, IL-23, and IL-1 $\beta$ ) and IL-17 or IL-22 levels.

Results are presented as ORs and CIs, which provide an accurate estimate of the relative risk.

1. Korn, T., Bettelli, E., Oukka, M., and Kuchroo, V.K 2009. IL-17 and Th17 cells. Annu. Rev. Immunol. 27:485-517.

2. Acosta-Rodriguez, E.V., et al. 2007. Surface phenotype and antigenic specificity of human interleukin 17-producing T helper memory cells. Nat. Immunol. 8:639-646.

3. Khader, S.A., et al. 2007. IL-23 and IL-17 in the establishment of protective pulmonary CD4+ T cell responses after vaccination and during Mycobacterium tuberculosis challenge. Nat. Immunol. 8:369-377.

4. Wolk, K., et al. 2004. IL-22 increases the innate immunity of tissues. Immunity. 21:241-254.

5. Wolk, K., and Sabat, R. 2006. Interleukin-22: a novel Tand NK-cell derived cytokine that regulates the biology of tissue cells. Cytokine Growth Factor Rev. 17:367-380.

6. Boniface, K., et al. 2005. IL-22 inhibits epidermal differentiation and induces proinflammatory gene expression and migration of human keratinocytes. J. Immunol. 174:3695-3702.

7. Ouyang, W., Kolls, J.K., and Zheng, Y. 2008. The biological functions of $T$ helper 17 cell effector cytokines in inflammation. Immunity. 28:454-467.

8. Ye, P., et al. 2001. Requirement of interleukin 17 receptor signaling for lung CXC chemokine and granulocyte colony-stimulating factor expression, neutrophil recruitment, and host defense. J. Exp. Med. 194:519-527.

9. Kelly, M.N., et al. 2005. Interleukin-17/interleukin-17 receptor-mediated signaling is important for generation of an optimal polymorphonuclear response against Toxoplasma gondii infection. Infect. Immun. 73:617-621.

10. Higgins, S.C., Jarnicki, A.G., Lavelle, E.C., and Mills, K.H. 2006. TLR4 mediates vaccine-induced protective cellular immunity to Bordetella pertussis: role of IL-17-producing T cells. J. Immunol. 177:7980-7989.

11. Zheng, Y., et al. 2008. Interleukin-22 mediates early host defense against attaching and effacing bacterial pathogens. Nat. Med. 14:282-289.

12. Scriba, T.J., et al. 2008. Distinct, specific IL-17- and IL-22-producing CD4+ T cell subsets contribute to the human anti-mycobacterial immune response. J. Immunol. 180:1962-1970.

13. Huang, W., Na, L., Fidel, P.L., and Schwarzenberger, P. 2004. Requirement of interleukin-17A for systemic anti-Candida albicans host defense in mice. J. Infect. Dis. 190:624-631.

14. Murray, H.W., Berman, J.D., Davies, C.R., and Saravia, N.G. 2005. Advances in leishmaniasis. Lancet. 366:1561-1577.

15. Hailu, A., et al. 2001. Distinct immunity in patients with visceral leishmaniasis from that in subclinically infected and drug-cured people: implications for the mechanism underlying drug cure. J. Infect. Dis. 184:112-115.

16. McFarlane, E., et al. 2008. Neutrophils contribute to development of a protective immune response

\section{Acknowledgments}

This work was funded by grants from INSERM and the French Ministry of Research. M.G.R. Pitta received a scholarship from the CAPES Foundation, the Ministry of Education, Brazil. A. Romano was funded by a scholarship from the French Ministry of Research.

Received for publication February 4, 2009, and accepted in revised form May 20, 2009.

Address correspondence to: Alain Dessein, Immunology and Genetics of Parasitic Diseases, INSERM UMR 906, Timone Faculty of Medicine, 27 Blvd. Jean Moulin, Marseille 13385, France. Phone: 33-4-91-32-44-53; Fax: 33-4-91-79-60-63; E-mail: alain. dessein@univmed.fr. during onset of infection with Leishmania donovani. Infect. Immun. 76:532-541.

17. Ribeiro-Gomes, F.L., et al. 2004. Macrophage interactions with neutrophils regulate Leishmania major infection. J. Immunol. 172:4454-4462.

18. Lima, G.M., et al. 1998. The role of polymorphonuclear leukocytes in the resistance to cutaneous Leishmaniasis. Immunol. Lett. 64:145-151.

19. Von Stebut, E. 2007. Immunology of cutaneous leishmaniasis: the role of mast cells, phagocytes and dendritic cells for protective immunity. Eur. J. Dermatol. 17:115-122.

20. Tacchini-Cottier, F., et al. 2000. An immunomodulatory function for neutrophils during the induction of a CD4+ Th2 response in BALB/c mice infected with Leishmania major. J. Immunol. 165:2628-2636.

21. Stager, S., et al. 2006. Distinct roles for IL-6 and IL-12p40 in mediating protection against Leishmania donovani and the expansion of IL-10+CD4+ T cells. Eur. J. Immunol. 36:1764-1771.

22. Ansari, N.A., Saluja, S., and Salotra, P. 2006. Elevated levels of interferon-gamma, interleukin-10, and interleukin- 6 during active disease in Indian kala azar. Clin. Immunol. 119:339-345.

23. Bacellar, O., et al. 1996. Interleukin-12 restores interferon-gamma production and cytotoxic responses in visceral leishmaniasis. J. Infect. Dis. 173:1515-1518.

24. Dong, C. 2008. TH17 cells in development: an updated view of their molecular identity and genetic programming. Nat. Rev. Immunol. 8:337-348.

25. Mangan, P.R., et al. 2006. Transforming growth factor-beta induces development of the $\mathrm{T}(\mathrm{H}) 17$ lineage. Nature. 441:231-234.

26. Veldhoen, M., Hocking, R.J., Atkins, C.J., Locksley, R.M., and Stockinger, B. 2006. TGFbeta in the context of an inflammatory cytokine milieu supports de novo differentiation of IL-17-producing T cells. Immunity. 24:179-189.

27. Locksley, R.M., et al. 1999. Susceptibility to infectious diseases: Leishmania as a paradigm. J. Infect. Dis. 179(Suppl. 2):S305-S308.

28. Langrish, C.L., et al. 2005. IL-23 drives a pathogenic $\mathrm{T}$ cell population that induces autoimmune inflammation. J. Exp. Med. 201:233-240.

29. Sutton, C., Brereton, C., Keogh, B., Mills, K.H., and Lavelle, E.C. 2006. A crucial role for interleukin (IL)- 1 in the induction of IL-17-producing T cells that mediate autoimmune encephalomyelitis. J. Exp. Med. 203:1685-1691.

30. Sonderegger, I., Kisielow, J., Meier, R., King, C., and Kopf, M. 2008. IL-21 and IL-21R are not required for development of Th17 cells and autoimmunity in vivo. Eur. J. Immunol. 38:1833-1838.

31. Coquet, J.M., Chakravarti, S., Smyth, M.J., and Godfrey, D.I. 2008. Cutting edge: IL-21 is not essential for Th17 differentiation or experimental autoimmune encephalomyelitis. J. Immunol. 180:7097-7101.
32. Annunziato, F., et al. 2007. Phenotypic and functional features of human Th17 cells. J. Exp. Med. 204:1849-1861.

33. Michel, M.L., et al. 2007. Identification of an IL-17producing NK1.1(neg) iNKT cell population involved in airway neutrophilia. J. Exp. Med. 204:995-1001.

34. Shibata, K., Yamada, H., Hara, H., Kishihara, K., and Yoshikai, Y. 2007. Resident Vdelta1+ gammadelta T cells control early infiltration of neutrophils after Escherichia coli infection via IL-17 production. J. Immunol. 178:4466-4472.

35. Ivanov, I.I., et al. 2006. The orphan nuclear receptor RORgammat directs the differentiation program of proinflammatory IL-17+ T helper cells. Cell. 126:1121-1133.

36. Hirota, K., et al. 2007. Preferential recruitment of CCR6-expressing Th17 cells to inflamed joints via CCL20 in rheumatoid arthritis and its animal model. J. Exp. Med. 204:2803-2812.

37. Liang, S.C., et al. 2006. Interleukin (IL)-22 and IL17 are coexpressed by Th 17 cells and cooperatively enhance expression of antimicrobial peptides. J. Exp. Med. 203:2271-2279.

38. Zenewicz, L.A., et al. 2007. Interleukin-22 but not interleukin-17 provides protection to hepatocytes during acute liver inflammation. Immunity. 27:647-659.

39. Happel, K.I., et al. 2003. Cutting edge: roles of Toll-like receptor 4 and IL-23 in IL-17 expression in response to Klebsiella pneumoniae infection. J. Immunol. 170:4432-4436.

40. Happel, K.I., et al. 2005. Divergent roles of IL-23 and IL-12 in host defense against Klebsiella pneumoniae. J. Exp. Med. 202:761-769.

41. Spahn, T.W., et al. 2008. CD4+ T cells transfer resistance against Citrobacter rodentium-induced infectious colitis by induction of Th 1 immunity. Scand. J. Immunol. 67:238-244.

42. Zheng, Y., et al. 2007. Interleukin-22, a T(H)17 cytokine, mediates IL-23-induced dermal inflammation and acanthosis. Nature. 445:648-651.

43. Yen, D., et al. 2006. IL-23 is essential for T cellmediated colitis and promotes inflammation via IL-17 and IL-6. J. Clin. Invest. 116:1310-1316.

44. Bucheton, B., et al. 2002. The interplay between environmental and host factors during an outbreak of visceral leishmaniasis in eastern Sudan. Microbes Infect. 4:1449-1457.

45. El-Safi, S.H., et al. 2002. Epidemiology of visceral leishmaniasis in Atbara River area, eastern Sudan: the outbreak of Barbar El Fugara village (19961997). Microbes Infect. 4:1439-1447.

46. Pratlong, F., et al. 2001. Sudan: the possible original focus of visceral leishmaniasis. Parasitology. 122:599-605.

47. Salhi, A., et al. 2008. Immunological and genetic evidence for a crucial role of IL-10 in cutaneous lesions in humans infected with Leishmania braziliensis. J. Immunol. 180:6139-6148. 